University of Nebraska - Lincoln

DigitalCommons@University of Nebraska - Lincoln

\title{
Polaron and Phonon Properties in Proton Intercalated Amorphous Tungsten Oxide Thin Films
}

\author{
M. Saenger \\ University of Nebraska - Lincoln \\ T. Hoing \\ Flabeg GmbH \& Co. KG, 93437 Furth im Wald, Germany \\ Brian W. Robertson \\ University of Nebraska - Lincoln, brobertson1@unl.edu \\ R. Billa \\ University of Nebraska - Lincoln \\ Tino Hofmann \\ University of Nebraska - Lincoln, thofmann4@unl.edu \\ See next page for additional authors
}

Follow this and additional works at: https://digitalcommons.unl.edu/cmrafacpub

Part of the Nanoscience and Nanotechnology Commons

Saenger, M.; Hoing, T.; Robertson, Brian W.; Billa, R.; Hofmann, Tino; Schubert, Eva; and Schubert, Mathias, "Polaron and Phonon Properties in Proton Intercalated Amorphous Tungsten Oxide Thin Films" (2008). Faculty Publications from Nebraska Center for Materials and Nanoscience. 88.

https://digitalcommons.unl.edu/cmrafacpub/88

This Article is brought to you for free and open access by the Materials and Nanoscience, Nebraska Center for (NCMN) at DigitalCommons@University of Nebraska - Lincoln. It has been accepted for inclusion in Faculty Publications from Nebraska Center for Materials and Nanoscience by an authorized administrator of DigitalCommons@University of Nebraska - Lincoln. 
Authors

M. Saenger, T. Hoing, Brian W. Robertson, R. Billa, Tino Hofmann, Eva Schubert, and Mathias Schubert 


\title{
Polaron and phonon properties in proton intercalated amorphous tungsten oxide thin films
}

\author{
M. F. Saenger, ${ }^{1, *}$ T. Höing, ${ }^{2}$ B. W. Robertson, ${ }^{3}$ R. B. Billa, ${ }^{3}$ T. Hofmann, ${ }^{1, *}$ E. Schubert, ${ }^{1, *}$ and M. Schubert ${ }^{1, *, \dagger}$ \\ ${ }^{1}$ Department of Electrical Engineering and Nebraska Center for Materials and Nanoscience, \\ University of Nebraska - Lincoln, Lincoln, Nebraska 68588, USA \\ ${ }^{2}$ Flabeg GmbH \& Co. KG, 93437 Furth im Wald, Germany \\ ${ }^{3}$ Department of Mechanical Engineering and Nebraska Center for Materials and Nanoscience, \\ University of Nebraska-Lincoln, Lincoln, Nebraska 68588, USA
}

(Received 24 August 2008; revised manuscript received 11 November 2008; published 22 December 2008)

\begin{abstract}
We report on the evolution of the polaron and phonon mode properties in amorphous tungsten oxide thin films measured by spectroscopic ellipsometry in the infrared to ultraviolet spectral regions as a function of the intercalated proton density. A parametric physical model dielectric function is presented, which excellently describes the ellipsometry data over a large intercalated charge-density range. Upon increased amounts of intercalated charge we observe a strong increase in the polaron absorption in the visible spectral range, a decrease in the infrared $\mathrm{W}-\mathrm{O}$ bond polarity, and an increase in the $\mathrm{W}=\mathrm{O}$ bond polarity. Our findings support the oxygen-extraction model as the polaron formation mechanism in tungsten oxide in agreement with previous theoretical works based on first-principles pseudopotential calculations. We discuss and suggest polaron formation by oxygen-related defect generation as origin for the coloration mechanism in tungsten oxide. We further discuss possible evidence for very large effective mass of the polarons within the insulator-to-metal transition regime.
\end{abstract}

DOI: 10.1103/PhysRevB.78.245205

PACS number(s): 78.20.-e

\section{INTRODUCTION}

Electrochromic materials change their optical properties upon intercalation of charge (coloration). The charge intercalation can be accompanied by oxidation processes. The change in optical properties is mostly assigned to formation of polarons and their contributions to the material's dielectric function (DF). A polaron is the combined lattice polarization charge induced by an excess charge carrier, which is surrounded and partially screened by the induced lattice charge. Spatial movement (polaron transport) of the excess charge carrier requires lattice distortion along the carrier path decorating the carrier with large effective mass and low mobility. ${ }^{1}$ Reversible electrochromic processes are very attractive for numerous applications. Electrochromic thin-film materials are employed in devices such as "smart windows," electrochromic rear view car mirrors, displays, emissivity modulation devices, and gas sensors, for example. ${ }^{2-5}$ The sixfoldcoordinated tungsten oxide $\left(\mathrm{WO}_{3}\right)$ is among the most investigated electrochromic materials and can be found in many applications. ${ }^{6-8}$ Despite large research efforts and numerous applications, the electronic mechanism responsible for the coloration process in $\mathrm{WO}_{3}$ is still not fully understood. It is unclear if the coloration in $\mathrm{WO}_{3}$ is associated with charge intercalation only or also with oxidation processes. It is further not clear which lattice sites and bonds are associated with the polaron formation.

$\mathrm{WO}_{3}$ is a wide band gap and highly insulating semiconductor with complex polymorphism and defect chemistry. ${ }_{3}-11$ In a simplified description, the crystal structure of $\mathrm{WO}_{3}$ is regarded as a modification of the perovskite-type $\mathrm{ABO}_{3}$ lattice, in which the $A$ site remains unoccupied and the $\mathrm{W}$ atoms occupy the $B$ site. This produces a three-dimensional network of corner-shared octahedra $\mathrm{WO}_{6} \cdot{ }^{9}{ }^{912-15}$ The tungsten oxide crystal structure is very unstable because the decrease in ion size of the $A$ site in the $A B X_{3}$ perovskite structure increases the instability of the structure, which is most unstable in $\mathrm{WO}_{3}$ because the ion for the $A$ site is missing. Preparation of amorphous tungsten oxide thin films is feasible with magnetron sputtering at room temperature and at convenient plasma conditions. ${ }^{11}$ The crystalline structure of tungsten oxide thin films strongly depends on the deposition temperature. In the case of $\mathrm{WO}_{3}$ as-deposited thin films fabricated at room temperature with magnetron sputtering, the crystalline structure is reported as amorphous from X-raydiffraction (XRD) measurements. ${ }^{16}$ Amorphous tungsten oxide films possess very low absorption in the visible spectral range and are therefore of interest for transparent metal oxide thin-film applications.

It is generally accepted that light absorption by polaron transitions (polaron absorption) is due to the transfer of electrons and the electron-induced lattice polarization clouds between adjacent tungsten ion sites. The polaron absorption mechanism is seen as the physical origin of the electrochromic property changes (electrochromism). ${ }^{17,18}$ The mechanism for producing polarons obviously must affect the local lattice bond properties. Accordingly, insertion or extraction of charge should be accompanied by distinct changes in the polarity of bond resonances; while at the same time the polaron absorption peak, typically seen in the near-infrared (IR) spectral range, must be detected. Likewise, for amorphous thin films, different bonds may be affected and new bonds may be created if the charge intercalation is further associated with chemical oxidation, for example. So far, quantitative investigations of polaron formation and associated lattice property changes upon charge intercalation in amorphous tungsten oxide films are missing. ${ }^{19}$ Evidence exists that upon coloration the oxidation number of the tungsten atoms reduces from $6+$ to $5+$ and which supports the formation of polarons. ${ }^{11}$ Tungsten atoms with fivefold oxidation numbers and their captured intercalated charge form po- 
larons. The trapped charge at the $\mathrm{W}^{5+}$ site can be transferred to a neighboring $\mathrm{W}^{6+}$ site by photon absorption. ${ }^{11,19}$ The absorption process is then interpreted by a hopping transfer mechanism of the polaron between neighboring tungsten atoms with different oxidation states. The photon energy is absorbed such that $\mathrm{W}^{6+}$ sites trap electrons and convert to the fivefold oxidation state. In addition, fourfold oxidation states may exist, which upon photon absorption may transform to fivefold oxidation by the same polaron hopping mechanism, but potentially with different excitation energy. ${ }^{20,21}$ Two plausible pictures exist for the actual formation of polarons: the double-charge intercalation model and the oxygenextraction model. ${ }^{22,23}$ In the double-charge intercalation model, a small cation (usually hydrogen, $\mathrm{H}^{+}, \mathrm{Li}^{+}$, or $\mathrm{Na}^{+}$) and electron are intercalated simultaneously, for example, into an amorphous tungsten oxide film. The positive ion is located at the $A$ site without forming bonds and acts as a charge compensation for the electron. The electron forms the polaron by changing the oxidation number of a $\mathrm{W}^{6+}$ site to $\mathrm{W}^{5+}$ and is able to transport ("hop") from one W site to another upon absorption of photons with sufficient energy. In contrast, the oxygen-extraction model suggests that oxygen atoms are extracted from the amorphous tungsten oxide network under the formation of water during an electrochemical process. ${ }^{22,23}$ The often observed columnar structure of amorphous tungsten oxide thin films could promote absorption of hydrogen ions by the porous film structure. After formation of $\mathrm{W}-\mathrm{O}-\mathrm{H}_{2}$, oxygen can be extracted and $\mathrm{H}_{2} \mathrm{O}$ is formed within a pore. The oxygen defect site then generates reduced tungsten sites which may further diffuse into the film while the corresponding electrons compensate for the oxygen loss forming polarons. The two pictures explain sufficiently polaron production and thus electrochromic alteration of the DF and cannot be distinguished from observation of the DF within the polaron absorption spectral region alone. Indirect support for the intercalation model was found from proton injection into $\mathrm{WO}_{3}$, where polaron absorption increased with increasing ion insertion. ${ }^{11,24}$ Yet it remained unclear whether the light ions were intercalated interstitially or if oxygen was also extracted from the amorphous tungsten oxide network. Interestingly, within the phonon (lattice) mode spectral absorption region the lattice vibrational properties should reflect different contributions to the DF within the two model pictures, since hydrogen incorporation (double-charge intercalation model) and oxygen extraction (extraction model) should affect the local lattice vibrational modes in different ways. So far, a precise measurement and analysis of the DF for tungsten oxide thin films within both the polaron and phonon absorption regions as a function of their intercalated charge density was not reported. Daniel et al. ${ }^{25}$ provided qualitative insight for the lattice resonance behavior in charge intercalated amorphous tungsten oxide films from polarized and unpolarized infrared reflectance data. However, because no quantitative DF model analysis of the complex thin-film spectra was attempted, the conclusions remained vague and confusion persisted about the correct interpretation of resonance features in the infrared spectra and the underlying phonon mode resonance frequencies. ${ }^{25}$

Here we report on the investigations of the DF of amorphous tungsten oxide films as a function of the intercalated proton $\left(\mathrm{H}^{+}\right)$density in the spectral range covering the IR, near-IR, visible (VIS), and ultraviolet (UV) spectral regions. Properties pertinent to other types of intercalation may not be the same as those discussed in this paper. We employ spectroscopic ellipsometry (SE) for determination of the DF from the IR to the UV spectral regions. IR spectroscopic ellipsometry (IRSE) has been demonstrated as a precise tool to determine phonon and free-charge-carrier properties of semiconductor thin films. ${ }^{26-29}$ Previous SE reports discussed the polaron absorption range from the near-IR to the VIS spectral range. ${ }^{11,19,30,31}$ Various approaches for physical lineshape analysis and polaron property assignment were employed such as oscillator approaches, ${ }^{31-34}$ and parametric semiconductor models with rigorous treatment of their density of state functions. ${ }^{33,35}$ Previous IRSE focused on uncolored amorphous tungsten oxide and electrochromic device performance for emittance modulation in the IR spectral region. ${ }^{4,36}$ Elucidation of the phonon mode properties in connection with the polaron properties is still lacking. Accordingly, no conclusive statement about the source of the polaron cause had been made. In this paper we employ physical line-shape model functions for parametrization of the measured DF over a large intercalated charge-density range. From our model analysis we assign the energies for the $\mathrm{W}^{4+} \rightleftharpoons \mathrm{W}^{5+}$ and the $\mathrm{W}^{5+} \rightleftharpoons \mathrm{W}^{6+}$ polaron transitions and discuss transition energy and oscillator amplitude parameters as a function of the intercalated charge density. In the IR range we observe changes in tungsten oxide phonon modes, which strongly depend on the intercalated charge density. We identify a distinct relation between the phonon and polaron properties, which sheds light on the source of the complex intercalation process. From our observations we conclude that strong indications are given in support of the oxygenextraction model. Furthermore, highly proton intercalated amorphous tungsten oxide films are investigated for possible evidence of a transition into a metal state, for which indications were found in electrical studies previously. ${ }^{37}$ Our lineshape analysis of the DF did not reveal the existence of a Drude term, which would indicate high densities of freecharge carriers. ${ }^{38,39}$ We discuss the absence of such contributions in light of possible insulator-to-metal transitions.

Our report is structured as follows. Section II outlines a brief overview on the ellipsometry technique and model DF approach, Sec. III describes the sample preparation and optical measurement procedure, and in Sec. IV the results are discussed. The paper closes with a brief summary of the results of this investigation.

\section{THEORY}

The DF of a material is a sensitive measure of the intrinsic electronic excitation mechanisms and reflects phonon, plasmon, exciton, and polaron properties, for example. ${ }^{1,39} \mathrm{~A}$ precise measurement of the DF over a sufficiently large spectral range and subsequent model line-shape analysis can identify the underlying physics of polarization and absorption processes. Spectroscopic ellipsometry is a powerful tool for precise measurement of the DF spectra from thin-film and bulk materials. ${ }^{40}$ In the following, we describe our SE 
data analysis procedure and the physical model line-shape functions to describe the DF of amorphous tungsten oxide films.

\section{A. SE data analysis}

$\mathrm{SE}$ is a versatile precise technique for the determination of the optical properties of surfaces, interfaces, and thin films. ${ }^{41}$ SE measures the ratio $\rho$ of the complex-valued Fresnel reflection coefficients for the polarizations perpendicular $R_{s}$ and parallel $R_{p}$ to the plane of incidence and is commonly expressed in terms of the ellipsometric parameters $\Psi$ and $\Delta$ (Refs. 40 and 42):

$$
\rho \equiv \frac{R_{p}}{R_{s}}=\tan (\Psi) e^{i \Delta} .
$$

The ellipsometric parameters depend on the thickness and DF $\varepsilon$ of the individual sample constituents. ${ }^{40}$ In order to extract the DF of the layer material of interest from the measured SE data, first a model has to be established, which consists of a sequence of parallel layers with perfectly abrupt interfaces and spatially homogeneous functions $\varepsilon$, bound between a semi-infinite substrate and the ambient. Then, a regression analysis (Levenberg-Marquardt parameter variation algorithm) is performed, where model parameters are varied until calculated and experimental data match as closely as possible. ${ }^{43}$ A direct way of obtaining the material's dielectric function of interest (in our case that of tungsten oxide) is to numerically invert the experimental SE data for each wavelength and independently of all other spectral data points (point-by-point analysis). This procedure requires in our case the knowledge of all layer thicknesses as well as the DF of all other sample constituents (electrode layer and substrate). The DF obtained from the point-by-point analysis needs to be further compared to physical line-shape models in order to obtain physically relevant parameters such as phonon mode frequencies and polaron parameters. ${ }^{44}$ The number of unknown parameters in the regression analysis can be reduced considerably if appropriate parametric-model lineshape functions are used to describe the DF for the layer material of interest. Thereby, all measured spectral data points are simultaneously involved in the regression analysis. In the case of a parametric-model analysis, the measured data are directly connected to the physical parameters of interest and, in principle, the layer thicknesses can also be obtained simultaneously. Furthermore, parametric models prevent wavelength-by-wavelength measurement noise from becoming part of the extracted DF, a possible error source for pointby-point analyzed spectra. ${ }^{39}$

\section{B. IR dielectric function model}

The phonon spectrum of crystalline $\mathrm{WO}_{3}$ corresponds to a bond-stretching $(\mathrm{W}-\mathrm{O})$ and an angle-bending mode $(\mathrm{O}-\mathrm{W}-$ O), which occur near 800 and $270 \mathrm{~cm}^{-1}$, respectively. ${ }^{45}$ In the case of amorphous materials no periodic ordering is present and therefore it is not possible to speak about a lattice; however, the amorphous network reveals specific vibration modes, which descends from the ideal crystal structure.
Polar lattice mode resonances cause distinct dispersion and absorption features in the spectral dependence of the DF. Each transverse-optic (TO) lattice mode has a corresponding longitudinal-optic (LO) mode. The so-called TO-LO rule requires that for multiple-phonon modes the frequencies of the TO and LO modes must alter according to a sequence "TO-LO-TO $\cdots$ LO." The frequencies of the TO and LO modes correspond to poles and zeros of the DF $\varepsilon(\omega)$, respectively. ${ }^{46,47}$ The amount of the TO-LO frequency splitting is a measure of the polar strength of each phonon branch. ${ }^{46,48,49}$ The contribution of " $\nu$ " polar lattice modes to the IR DF at photon energy $\hbar \omega$ can be described using a set of Lorentz oscillators with anharmonic broadening to account for the coupling between the TO and LO modes in multiple-phonon mode materials, ${ }^{50}$

$$
\varepsilon^{\mathrm{ph}}(\omega)=\varepsilon_{\infty} \prod_{s=1}^{\nu} \frac{\omega^{2}+i \gamma_{\mathrm{LO}, s} \omega-\omega_{\mathrm{LO}, s}^{2}}{\omega^{2}+i \gamma_{\mathrm{TO}, s} \omega-\omega_{\mathrm{TO}, s}^{2}},
$$

where $\omega_{\mathrm{LO}, s}, \gamma_{\mathrm{LO}, s}, \omega_{\mathrm{TO}, s}, \gamma_{\mathrm{TO}, s}$, and $\varepsilon_{\infty}$ are the wave number and broadening values of the sth LO and TO phonon mode, and the high-frequency limit, respectively, and where the index $s$ runs over $\nu$ modes $(i=\sqrt{-1}$ denotes the imaginary unit). To keep the physical meaning of $\varepsilon(\omega)$ in Eq. (2), the condition $\operatorname{Im}\{\varepsilon(\omega)\} \geq 0, \forall \omega$, the TO-LO rule and the generalized Lowndes condition must be satisfied. ${ }^{38,39,51}$

\section{Polaron contribution}

Amorphous tungsten oxide films possess a roomtemperature band-gap energy of $\sim 3.4 \mathrm{eV}$, whereas the bandgap energy for monoclinic crystalline material was found at $2.6 \mathrm{eV} .{ }^{11,52,53}$ In the present study only amorphous tungsten oxide films are investigated and the below-band-gap spectral region is considered. According to the frequency-dependent electrical conductivity theory of Reik and Heese ${ }^{54}$ and Schirmer et al. ${ }^{55}$ the polaron contribution to the DF is described by a single Gaussian line shape or alternatively by a harmonic Lorentzian-broadened oscillator. ${ }^{2,32,56,57}$ It has already been observed that a single Gaussian or Lorentzian line shape is not fully sufficient to describe the experimentally observed absorption behavior. ${ }^{33,55,58,59}$ Here we obtain that polaron-induced DF changes in colored tungsten oxide film must be modeled by two separate oscillators with Lorentzian and Gaussian line shapes. We identify these oscillators with the transitions between $\mathrm{W}^{4+}$ and $\mathrm{W}^{5+}$ and between $\mathrm{W}^{5+}$ and $\mathrm{W}^{6+}$ states, respectively, as described below. The polaron contribution to $\varepsilon$ used here reads as

$$
\begin{aligned}
\varepsilon^{P}(\hbar \omega)= & \frac{A^{(4-5)} E_{P}^{(4-5)} \gamma}{\left(E_{P}^{(4-5)}\right)^{2}-(\hbar \omega)^{2}+i \gamma^{(4-5)} \hbar \omega} \\
& +A^{(5-6)} \exp \left[-\left(\frac{\hbar \omega-E_{P}^{(5-6)}}{i \gamma^{(5-6)}}\right)^{2}\right],
\end{aligned}
$$

where $A^{(\cdots)}, E_{P}^{(\cdots)}$, and $\gamma^{(\cdots)}$ correspond to the amplitude, polaron transition energy, and oscillator broadening parameters, respectively. The transitions $\mathrm{W}^{4+} \rightleftharpoons \mathrm{W}^{5+}$ and $\mathrm{W}^{5+} \rightleftharpoons \mathrm{W}^{6+}$ are denoted by " $4-5$ " and "5-6" within the superscript, respectively. 


\section{Higher electronic transitions}

In addition to the phonon and polaron contributions, within the near-band-gap region the DF of amorphous tungsten oxide further contains contributions from higher-energy electronic transitions, including the band-gap transition itself. Their contributions for photon energies below the bandgap energy can be properly described by the empirical Cauchy relation, ${ }^{32}$

$$
\varepsilon^{C}(\hbar \omega)=\left[C_{0}+C_{1}(\hbar \omega)^{2}+C_{2}(\hbar \omega)^{4}\right]^{2},
$$

where $C_{i}$ are the Cauchy model parameters.

The model DF used in this work is the sum of all individual contributions,

$$
\varepsilon=\varepsilon^{\mathrm{ph}}+\varepsilon^{P}+\varepsilon^{C} .
$$

\section{EXPERIMENT}

A set of equally sized samples was deposited under identical growth conditions at room temperature by radio frequency magnetron sputtering in a large-scale industrial deposition system. Except for one sample, prior to the 270-nmthick tungsten oxide films, an indium tin oxide (ITO) layer of thickness $170 \mathrm{~nm}$ was grown on the glass substrate. The ITO film served as working electrode during the electrochemical preparation of the samples, whereas the counterelectrode and the reference electrode consisted of a $99.9 \%$ pure titanium plate and an $\mathrm{Ag}-\mathrm{Ag}$ calomel, respectively. The charge density in the samples was controlled by a potentiostat. We used an electrolyte consisting of a $0.11 \mathrm{CF}_{3} \mathrm{COOH}$ and $0.4 \mathrm{TBAClO}_{4}$ molar solution in propylene carbonate, with hydrogen ions $\left(\mathrm{H}^{+}\right)$as charge carriers. The inserted ion density per surface area was obtained from the samples surface area $A$ immersed into the electrolyte and the total charge $Q$.

The ratio $x$ of intercalated charge per tungsten ion was calculated using the molar equation for the number of tungsten ions per volume,

$$
x=\frac{Q M}{e A d \varrho N_{A}},
$$

where $M$ is the molar mass of tungsten oxide, $d$ is the thickness, $\varrho\left(\mathrm{g} / \mathrm{cm}^{3}\right)$ is the film density, $e$ is the elementary charge, and $N_{A}$ is Avogadro's constant. For the present calculations the values for the thickness and mass density were $d=270 \mathrm{~nm}$ and $\varrho=3.6 \mathrm{~g} / \mathrm{cm}^{3}$. The mass density is a reported typical value for amorphous tungsten oxide films, as determined by Rutherford backscattering spectrometry and $\mathrm{x}$-ray diffraction. ${ }^{31}$ To ensure an identical initial state for all samples, prior to the coloration all samples were bleached by applying a voltage of $2 \mathrm{~V}$ for $5 \mathrm{~min}$ to the ITO working electrode and optically characterized. Thereafter, each sample was charged to a different charge-ion ratio $x$ in the range from 0 to 0.5 , which corresponds to a coloration range from bleached to $\sim 57 \%$ of the maximal coloration as estimated from the saturation model discussed below. Further increase in the intercalated charge was not attempted to avoid irreversible changes.

Ellipsometric data in the near-IR to UV spectral range (from 0.73 to $3.34 \mathrm{eV}$ ) were acquired with a rotating com-

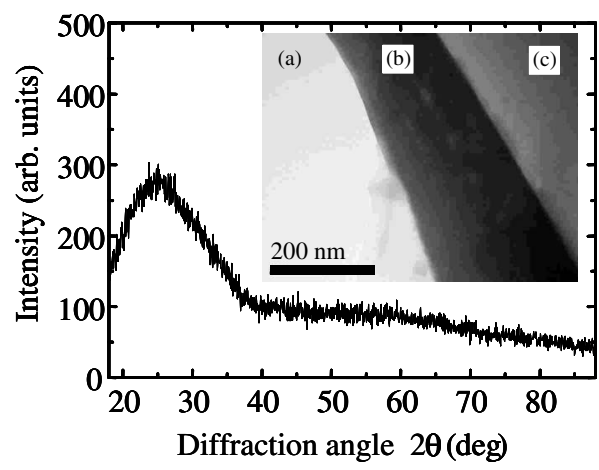

FIG. 1. $\mathrm{Cu} K \alpha$ diffraction pattern of as-deposited $\mathrm{WO}_{3}$ on glass. The inset depicts a bright field TEM microphotograph of the sample cross section, where (a) the void, (b) the $\mathrm{WO}_{3}$ film, and (c) the glass substrate can be observed.

pensator ellipsometer (M-2000 ${ }^{\mathrm{TM}}$, J. A. Woollam Co.) at normal incidence for transmission and at angles of incidence $\Phi=60^{\circ}, 65^{\circ}, 70^{\circ}$, and $75^{\circ}$. In the mid-IR (from 300 to $5000 \mathrm{~cm}^{-1}$ ), a Fourier-transform-based spectroscopic ellipsometer (IR-VASETM, J. A. Woollam Co.) was employed. In order to reduce the measurement time, the measurements for the intercalated samples were carried out at $\Phi=70^{\circ}$ only. All optical measurements were performed in a continuously purged $\mathrm{N}_{2}$ atmosphere and at room temperature, where the instruments were placed inside a dry chamber. Normalincidence unpolarized transmission intensity measurements in the photon energy range of $0.73-3.34 \mathrm{eV}$ were performed. The crystallographic structure of the investigated $\mathrm{WO}_{3}$ thin films here was analyzed by XRD with a high-resolution diffractometer (D/Max-B ${ }^{\mathrm{TM}}$, Rigaku Co.). $\mathrm{Cu} K \alpha$ radiation was used for the diffraction experiments. The XRD spectra were acquired with a scanning step resolution of $0.05^{\circ}$. For the TEM investigation, cross-section sample specimens were prepared out from the $\mathrm{WO}_{3}$ coated glass sample. Core disks were drilled from the sample. The glass was mechanically dimpled down to ca. $5 \mu \mathrm{m}$. The final thinning was done on a dual argon-ion gun milling system from the side of the glass only. The microphotographs were performed using a Jeol 2010 high-resolution transmission electron microscope operating $200 \mathrm{kV}$.

\section{RESULTS AND DISCUSSION}

\section{A. Structural properties}

The XRD pattern of as-deposited $\mathrm{WO}_{3}$ on glass is shown in Fig. 1. The diffractogram presents the typical XRD pattern of amorphous $\mathrm{WO}_{3}$ on glass as observed in the literature. ${ }^{16}$ The inset shows the TEM microphotograph of the film where a uniform structure without any domain boundaries can be observed.

\section{B. Polaron properties}

Figure 2 shows the experimental and best-match model calculated data for the ellipsometric parameters $\Psi$ and $\Delta$ in the near-IR to UV spectral range obtained for five samples 


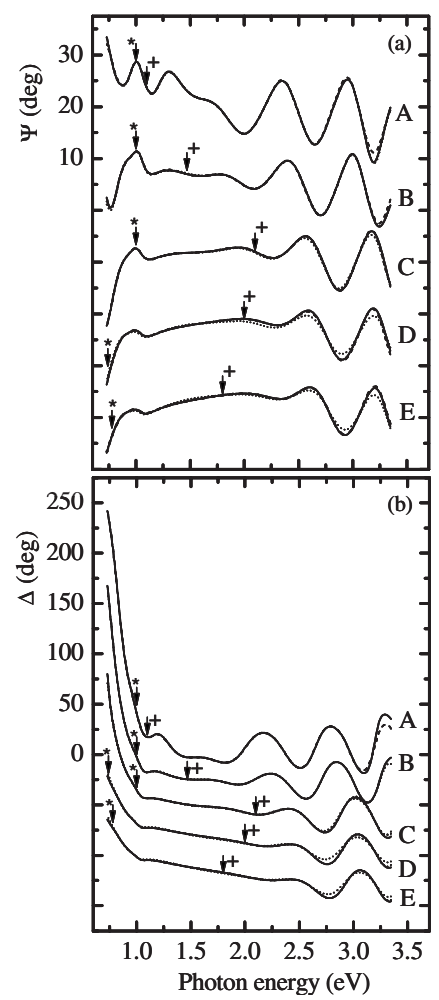

FIG. 2. Near-IR to UV ellipsometric parameters (a) $\Psi$ and (b) $\Delta$ for amorphous tungsten oxide films on ITO/glass with increasing intercalated charge densities (A: $x=0$; B: $x=0.124$; C: $x=0.250$; D: $x=0.375$; and $\mathrm{E}: x=0.5$ ). Spectra are shifted with respect to data $\mathrm{A}$ for better visualization. Data are indicated by solid lines for experiment, dashed lines for double (Gauss-Lorentz) oscillator approach, and dotted lines for single Lorentz oscillator approach. Asterisk and cross denote energies of the $\mathrm{W}^{4+} \rightleftharpoons \mathrm{W}^{5+}$ and $\mathrm{W}^{5+} \rightleftharpoons \mathrm{W}^{6+}$ transitions, respectively.

with charge densities $x$ from 0 to 0.5 . Each sample was measured before and after coloration. The data prior to coloration for samples B-E are identical with those shown for sample A in Fig. 2. Figure 3(a) depicts the normal-incidence transmission spectra for the same samples. Fabry-Pérot oscillations dominate the spectra of the noncharged sample. The gradual disappearance of Fabry-Pérot oscillations in the visible range with increasing intercalated charge densities is caused by the gradual appearance of strong absorption in the near-IR to VIS spectral range within the amorphous tungsten oxide films and which is due to the polaron formation upon charge intercalation. A five-phase model [glass substrate/ITO layer/ tungsten oxide $(d) /$ surface roughness $\left(d_{\text {rough }}\right) /$ ambient] was employed for analysis of the $\Psi$ and $\Delta$ spectra from each sample and subsequent comparison with the transmission data. We accounted for the (minor) effect of roughness at the tungsten oxide/ambient interface by including a dielectric layer $\left(d_{\text {rough }} \approx 7 \mathrm{~nm}\right)$. The Bruggeman effective-medium approximation (EMA) was employed to mimic the DF of this thin overlayer mixing the DF of amorphous tungsten oxide and voids in equal parts. The optical response of the amorphous tungsten oxide film was treated as that of a homogeneous isotropic material, while the ITO layer was described following the previous work from Synowicki. ${ }^{60}$

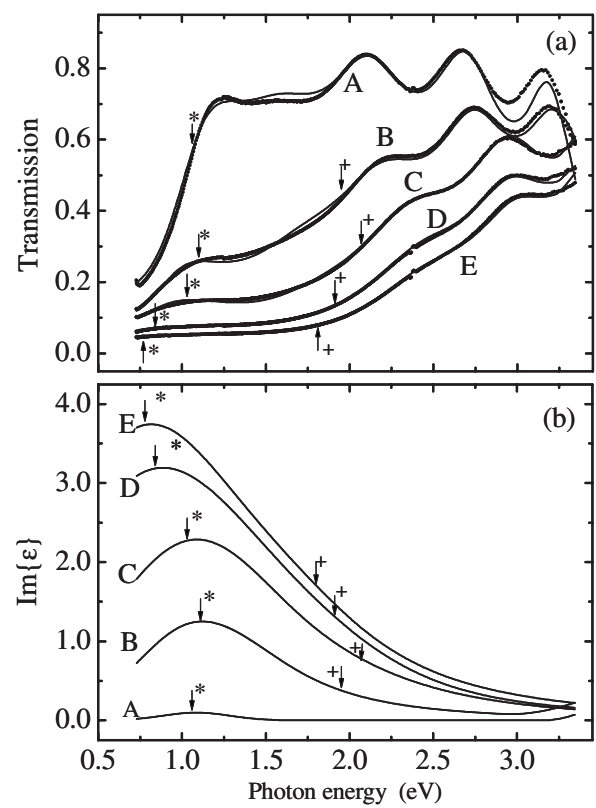

FIG. 3. (a) Normal-incidence transmission intensity spectra for the same samples in Fig. 2. (b) Best-model calculated $\operatorname{Im}\{\varepsilon\}$ of the model DF in the polaron region obtained from data analysis in Fig. 2 and (a). Vertical arrows with asterisk and cross indicate the photon energy of the $\mathrm{W}^{4+} \rightleftharpoons \mathrm{W}^{5+}(4-5)$ and $\mathrm{W}^{5+} \rightleftharpoons \mathrm{W}^{6+}$ (5-6) polaron transitions, respectively.

Figure 3(b) presents the best-match model calculated near-IR to UV model DF for the differently charged amorphous tungsten oxide films. The Lorentz-Gaussian doubleoscillator model in Eq. (3) was used for parametrization of the model DF. The main contribution of the absorption is described by the Gaussian line shape, which reveals a higherenergy side band modeled by the Lorentz oscillator. The match between experiment (solid lines) and model calculation (dashed lines) leads to virtually indistinguishable graphs in Fig. 2. We also attempted to use a single Lorentz oscillator model (dotted lines in Fig. 2) and observed increasing deviation between experiment and model data for higher charged samples, which are included in Fig. 2 as well. The doubleoscillator description implies the presence of two separate optical transitions. This finding is corroborated by the model suggested previously by Lee et al. $^{61}$ Based on Raman measurements of $\mathrm{Li}^{+}$-intercalated $\mathrm{WO}_{3}$ thin films, Lee et al. ${ }^{61}$ concluded that as-deposited amorphous $\mathrm{WO}_{3}$ thin films include predominantly $\mathrm{W}^{6+}$ and $\mathrm{W}^{4+}$ states denoted as $\mathrm{W}_{1-x}^{6+} \mathrm{W}^{4+} \mathrm{O}_{3-x}$. The charge intercalation leads to a reduction in a fraction of the $\mathrm{W}^{6+}$ states to $\mathrm{W}^{5+}$ while leaving the $\mathrm{W}^{4+}$ states unaffected. The colored films therefore contain $\mathrm{W}^{4+}$, $\mathrm{W}^{5+}$, and $\mathrm{W}^{6+}$ states, and the optical absorption is caused by optical polaron transitions between $\mathrm{W}^{4+}$ and $\mathrm{W}^{5+}$ states $\left(\mathrm{W}^{4+} \rightleftharpoons \mathrm{W}^{5+}\right)$ and $\mathrm{W}^{5+}$ and $\mathrm{W}^{6+}$ states $\left(\mathrm{W}^{5+} \rightleftharpoons \mathrm{W}^{6+}\right)$,

$$
\begin{gathered}
h \nu+\mathrm{W}_{i}^{4+}+\mathrm{W}_{j}^{5+} \rightarrow \mathrm{W}_{i}^{5+}+\mathrm{W}_{j}^{4+}, \\
h \nu^{\prime}+\mathrm{W}_{i}^{5+}+\mathrm{W}_{j}^{6+} \rightarrow \mathrm{W}_{i}^{6+}+\mathrm{W}_{j}^{5+},
\end{gathered}
$$

where $h \nu$ and $h \nu^{\prime}$ are the corresponding photon energies. Ozkan et al..$^{21}$ concluded previously from optical-absorption 


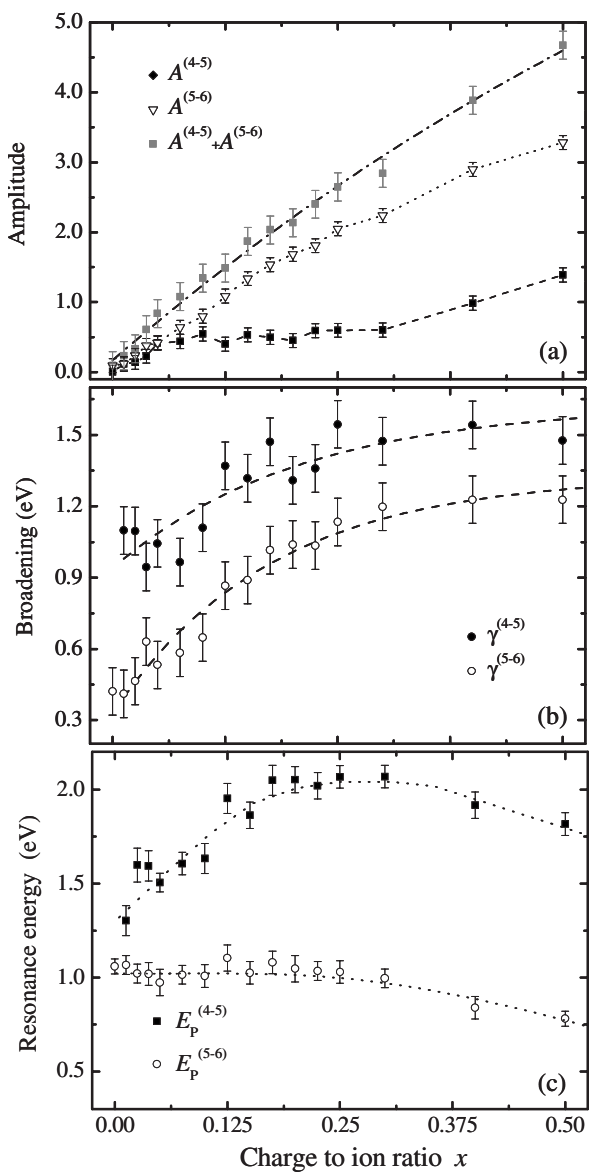

FIG. 4. (a) Double-oscillator amplitude parameters for the 4-5 and 5-6 polaron transitions as a function of the charge intercalation. (b) Same as (a) for the broadening parameters. (c) Polaron energy parameters. The dash-dotted line in (a) is the best-match model curve following the exponential saturation behavior. Dashed and dotted lines are plotted as guides for the eyes.

measurements that the $\mathrm{W}^{4+} \rightleftharpoons \mathrm{W}^{5+}$ transition occurs at 1.75 $\mathrm{eV}$, whereas the $\mathrm{W}^{5+} \rightleftharpoons \mathrm{W}^{6+}$ transition was found at $1 \mathrm{eV}$. Both transition energies are in close proximity to the energies found here from our double-oscillator model and which are indicated in Figs. 2 and 3(b). Reik and Heese ${ }^{54}$ and Schirmer et $a l .{ }^{55}$ described the polaron contribution to the DF by a single Gaussian line shape. Description of the polaron contribution with a single Lorentz oscillator was also often reported. 5,31,32,57 However, many authors indicated that a single Gaussian line shape cannot completely account for the optical properties of colored $\mathrm{WO}_{3} \cdot{ }^{33,55,58,59}$ Our analysis reveals that the polaron-induced DF changes in colored amorphous tungsten oxide films is excellently characterized for charge densities between $x=0, \ldots, 0.5$ by using two separate oscillators with Lorentzian and Gaussian line shapes. We observed upon implementation of the assignment by Ozkan et $a l{ }^{21}$ that the Lorentz oscillator accounts for the $\mathrm{W}^{4+} \rightleftharpoons \mathrm{W}^{5+}$ transition and that the Gaussian is associated with the $\mathrm{W}^{5+} \rightleftharpoons \mathrm{W}^{6+}$ transition.

Figure 4(a) summarizes the dependencies of the amplitude parameters of the $\mathrm{W}^{4+} \rightleftharpoons \mathrm{W}^{5+}$ and $\mathrm{W}^{5+} \rightleftharpoons \mathrm{W}^{6+}$ transitions for different intercalated charge densities. Both amplitude parameters increase upon charge intercalation. Here we have included results from ten additional samples with various charge densities. It is of interest to note that the two amplitudes reflect a competing relationship. While the individual amplitudes vary nonexponentially, the sum of both amplitudes can be well described by an exponential saturation behavior,

$$
A(x)=A_{1}\left(1-e^{-k_{A} x}\right)+A_{0} .
$$

The best-match calculation using Eq. (8) to the sum of the amplitude parameters as a function of the charge density results in $A_{1}=11.3 \pm 2.6, \quad A_{0}=0.173 .3 \pm 0.05$, and $k_{A}$ $=0.99 \pm 0.28$. This saturation behavior is typical for the coloration of tungsten oxide. ${ }^{32,62,63}$ The increase in absorption and exponential saturation with increase in charge density $x$ can be well understood by polaron hopping mechanisms. As the number of the intercalated electrons (charge density $x$ ) increases, the number of "empty" sites $\left(n_{\text {emp }}\right)$ reduces. Assuming that the optical polaron absorption takes place if an empty site is available and that this absorption is proportional to the number of intercalated charge $\left(1-n_{\mathrm{emp}}\right)$, the problem reduces to finding $n_{\mathrm{emp}}$. If the change in $n_{\mathrm{emp}}$ with charge density $x$ is proportional to $x$ then

$$
\frac{d n_{\mathrm{emp}}}{d x}=-k_{\alpha} x,
$$

and $n_{\mathrm{emp}}=N e^{-k_{\alpha} x}$, where $N$ is the total number of available sites. If every polaron transition contributes to the absorption by $\Delta \alpha$ then the absorption can be expressed as

$$
\alpha(x)=\alpha_{\max }\left(1-e^{-k_{\alpha} x}\right),
$$

where $\alpha_{\max }=\Delta \alpha N$. Indeed, the observed DF model amplitude parameters follow such exponential behavior. Equation (8) may be used to estimate the coloration in our samples, which apparently reached $\sim 41 \%$ according to the maximum combined amplitude for $x=0.5$ in Fig. $4 .{ }^{64}$

Figure 4(b) depicts the broadening parameters of the $\mathrm{W}^{4+} \rightleftharpoons \mathrm{W}^{5+}$ and $\mathrm{W}^{5+} \rightleftharpoons \mathrm{W}^{6+}$ transition as a function of the intercalated charge density. Both parameters initially increase with charge density and then seem to saturate; however, both also carry a large uncertainty limit. Figure 4(c) presents the dependencies of the energy parameters of the $\mathrm{W}^{4+} \rightleftharpoons \mathrm{W}^{5+}$ and $\mathrm{W}^{5+} \rightleftharpoons \mathrm{W}^{6+}$ transitions. As the charge density increases, for small densities, the $\mathrm{W}^{5+} \rightleftharpoons \mathrm{W}^{6+}$ transition energy remains constant, while the $\mathrm{W}^{4+} \rightleftharpoons \mathrm{W}^{5+}$ transition is strongly blue shifted. At $x \sim 0.25$ both transitions begin to be affected by a redshift. The redshift of the resonance energy with increasing charge densities is concordant with observations made in previous investigations on tungsten oxide film samples. ${ }^{33,65,66}$ The redshift is caused by the increasing many-body charge interaction and indicates the gradual transition to the metallic state (insulator-to-metal transition) in tungsten oxide. The initial strong blueshift of the $\mathrm{W}^{4+} \rightleftharpoons \mathrm{W}^{5+}$ transition remains unexplained at this point.

\section{Mid-IR spectral range}

The ellipsometric data were analyzed using a model layer calculation scheme analogous to the data analysis for the near-IR to UV spectral regions. Figure 5 shows the experi- 


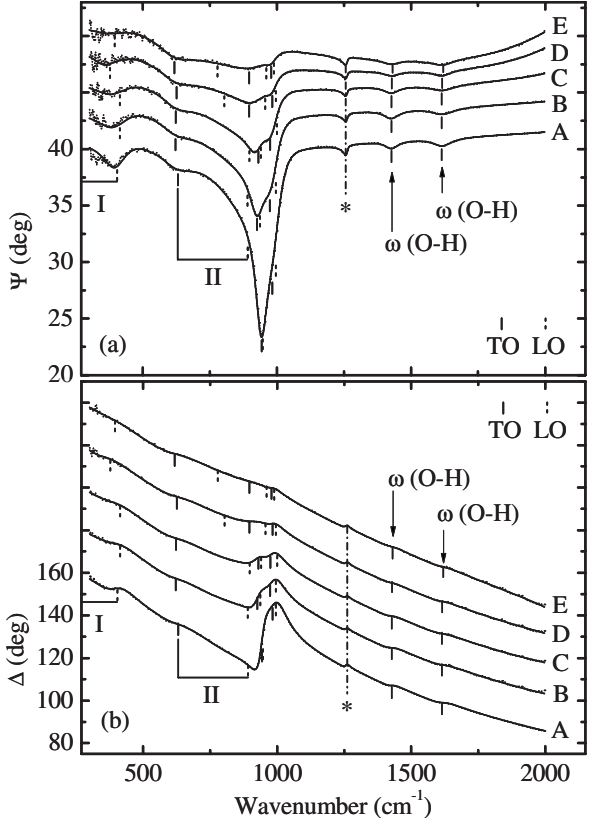

FIG. 5. Experimental (dotted lines) and best-match calculated (solid lines) SE $\Psi$ and $\Delta$ spectra at an angle of incidence of $70^{\circ}$. The charge densities are the same as in Fig. 2. Brackets indicate TO (solid vertical lines) and LO (dashed vertical lines) of tungsten oxide bending (I) and stretching (II) modes. The $\Psi(\Delta)$ spectra are shifted in steps of $2.5^{\circ}\left(20^{\circ}\right)$ with respect to data set A for better visualization. The hydroxyl group vibrations are indicated by vertical arrows.

mental and best-match model calculated $\Psi$ and $\Delta$ IRSE spectra, where charge concentration increases from bottom to top. Both experimental data and best-match model are virtually indistinguishable. The IRSE data in the measured spectral range of interest from 300 to $2000 \mathrm{~cm}^{-1}$ reveal strong contributions from lattice vibration modes. In addition, depending on the intercalated charge density $x$, a subtle lowenergy tail of the polaron contribution extends into the IR spectral region. The asterisk in Fig. 5 marks a feature that originates from the underlying glass optical properties. Figures 6 and 7 present $\operatorname{Im}\{\varepsilon\}$ and $\operatorname{Im}\{-1 / \varepsilon\}$, respectively, obtained by the parametrized model DF approach (solid lines) and the point-by-point data inversion (dotted lines). ${ }^{67}$ Both approaches were described in detail previously. ${ }^{44}$ Table I summarizes the best-match IR DF model parameters. We emphasize the excellent agreement between the point-bypoint inverted DF spectra and the model DF. Model lineshape approaches can subsume small resonance lines into adjacent model line shapes of larger amplitudes. Subtle features may thereby become extinct within the resulting spectra, when presented as the "original" data. The differences between the point-by-point inversion and the model DF spectra in Figs. 6 and 7 are minor only and represent data noise.

The IR model DF in Figs. 6 and 7 for amorphous tungsten oxide films requires contributions from six oscillators according to Eq. (2). Two oscillators account for the bending (labeled band I in Fig. 8) and stretching (band II in Fig. 8) lattice vibration modes and two modes for $\mathrm{W}=\mathrm{O}$ bonds (la-

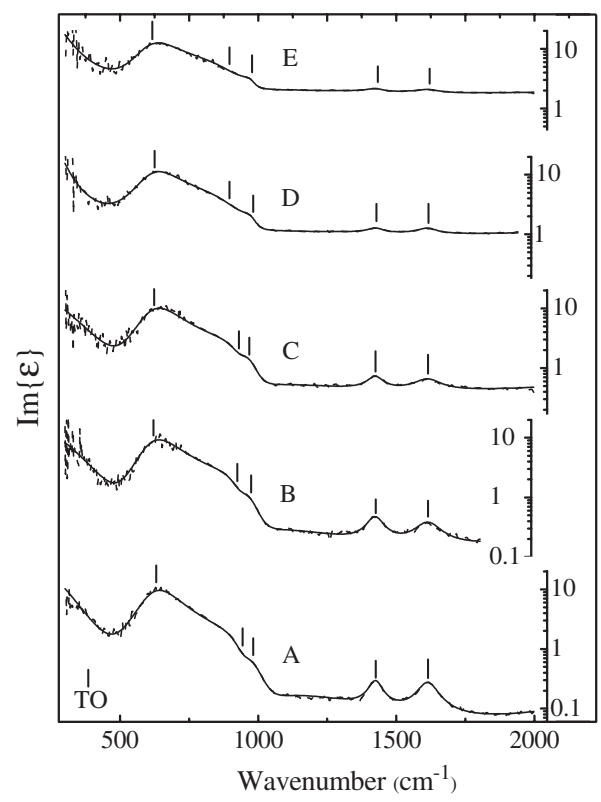

FIG. 6. Point-by-point inverted (dotted lines) and model DF (solid lines) $\operatorname{Im}\{\varepsilon\}$ spectra. Vertical lines indicate TO (solid line) mode parameters within the model DF.

beled III and IV in Fig. 8). Two additional harmonic oscillators [Eq. (2) with $\gamma_{\mathrm{TO}}=\gamma_{\mathrm{LO}}$ ] are required for wave numbers $\omega \sim 1420$ and $\omega \sim 1620 \mathrm{~cm}^{-1}$. These frequencies coincide with hydroxyl $(\mathrm{O}-\mathrm{H})$ group vibrations. A subtle polaroninduced IR absorption tail was included by the doubleoscillator model functions and parameters corresponding to the actual intercalated charge concentration as discussed in Sec. IV B. TO (solid lines) and LO (dashed lines) vibration modes amorphous tungsten oxide identified here are indicated by vertical lines in Figs. 5-7.

Figure 8 summarizes the TO and LO frequencies versus intercalated charge density. Hatched areas indicate the re-

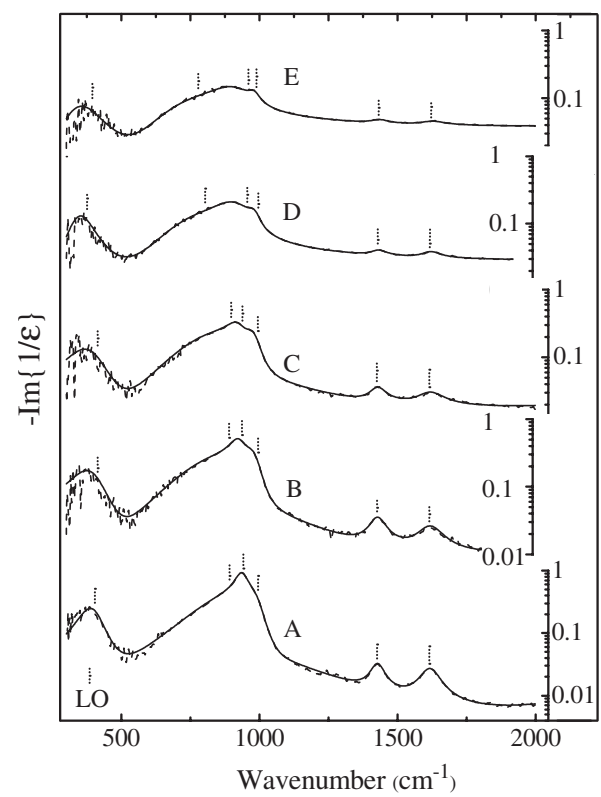

FIG. 7. Same as Fig. 6 for $\operatorname{Im}(-1 / \varepsilon)$. Vertical lines indicate LO-mode parameter frequencies obtained from the model DF. 
TABLE I. Best-match model calculation lattice vibration mode parameters in $\left(\mathrm{cm}^{-1}\right)$ at various intercalated charge densities. The confidence limits are given in parentheses.

\begin{tabular}{|c|c|c|c|c|}
\hline$x$ & $\omega_{\mathrm{TO}, \mathrm{I}}$ & $\omega_{\mathrm{LO}, \mathrm{I}}$ & $\omega_{\mathrm{TO}, \mathrm{II}}$ & $\omega_{\mathrm{LO}, \mathrm{II}}$ \\
\hline \multirow[t]{2}{*}{0.0} & 278.5 & 403.9 & 631.1 & 890.9 \\
\hline & (4.6) & $(1.7)$ & (1.5) & (2.9) \\
\hline \multirow[t]{2}{*}{0.124} & 278.5 & 414.1 & 621.4 & 890.4 \\
\hline & (4.6) & $(4.0)$ & (3.8) & $(7.3)$ \\
\hline \multirow[t]{2}{*}{0.250} & 278.5 & 415.8 & 623.8 & 897.3 \\
\hline & $(4.6)$ & $(5.6)$ & $(4.2)$ & (10.9) \\
\hline \multirow[t]{2}{*}{0.375} & 278.5 & 375.7 & 625.5 & 803 \\
\hline & (4.6) & $(12.5)$ & $(6.1)$ & (29) \\
\hline \multirow[t]{2}{*}{0.50} & 278.5 & 397 & 614.0 & 781 \\
\hline & $(4.6)$ & (11) & $(7.7)$ & (60) \\
\hline$x$ & $\omega_{\mathrm{TO}, \mathrm{III}}$ & $\omega_{\mathrm{LO}, \mathrm{III}}$ & $\omega_{\mathrm{TO}, \mathrm{IV}}$ & $\omega_{\mathrm{LO}, \mathrm{IV}}$ \\
\hline \multirow[t]{2}{*}{0.0} & 942.1 & 943.5 & 981.5 & 995.2 \\
\hline & (2.0) & $(0.3)$ & $(2.4)$ & $(0.5)$ \\
\hline \multirow[t]{2}{*}{0.124} & 924.6 & 937.1 & 973.9 & 995.1 \\
\hline & (4.6) & $(1.5)$ & (3.6) & $(0.7)$ \\
\hline \multirow[t]{2}{*}{0.250} & 930.6 & 939.5 & 968.1 & 1000.1 \\
\hline & $(9.3)$ & $(3.7)$ & $(6.3)$ & $(1.3)$ \\
\hline \multirow[t]{2}{*}{0.375} & 896 & 956.8 & 982.1 & 995.9 \\
\hline & (15) & $(6.7)$ & $(3.1)$ & $(2.1)$ \\
\hline \multirow[t]{2}{*}{0.5} & 896 & 955 & 978.4 & 988.9 \\
\hline & (22) & (11) & (2.1) & (1.7) \\
\hline$x$ & $\omega_{\mathrm{TO}, \mathrm{V}}$ & $\omega_{\mathrm{LO}, \mathrm{V}}$ & $\omega_{\mathrm{TO}, \mathrm{VI}}$ & $\omega_{\mathrm{LO}, \mathrm{VI}}$ \\
\hline \multirow[t]{2}{*}{0.0} & 1425.6 & 1427.6 & 1614.6 & 1617.6 \\
\hline & $(0.7)$ & $(0.7)$ & $(0.7)$ & $(0.7)$ \\
\hline \multirow[t]{2}{*}{0.124} & 1424.1 & 1428.0 & 1614.7 & 1619.0 \\
\hline & $(0.9)$ & $(0.9)$ & $(1.4)$ & $(1.5)$ \\
\hline \multirow[t]{2}{*}{0.250} & 1425.3 & 1428.9 & 1614.7 & 1619.6 \\
\hline & $(1.3)$ & $(1.3)$ & $(2.0)$ & $(2.1)$ \\
\hline \multirow[t]{2}{*}{0.375} & 1429.4 & 1431.9 & 1617.1 & 1620.3 \\
\hline & $(1.5)$ & $(1.5)$ & $(1.3)$ & $(1.3)$ \\
\hline \multirow[t]{2}{*}{0.50} & 1431.0 & 1433.9 & 1621.6 & 1625.5 \\
\hline & $(1.7)$ & $(1.6)$ & $(1.8)$ & (1.8) \\
\hline
\end{tabular}

gions between individual TO-LO pairs and which correspond to bands of high reflectivity. The TO mode for band I is outside the spectral range accessible here. Therefore, its frequency was assumed from previous studies and not further varied during data analysis. The main observations from the evolution of the vibrational modes with intercalated charge density are that (i) band II diminishes drastically with $x$, (ii) bands III and IV evolve and increase with $x$, and (iii) the hydroxyl group vibrations remain mostly unaffected by $x$.

\section{Bands I and II}

The TO frequency of band II remains unaffected, while the LO mode clearly shows a redshift for $x>0.375$. This is

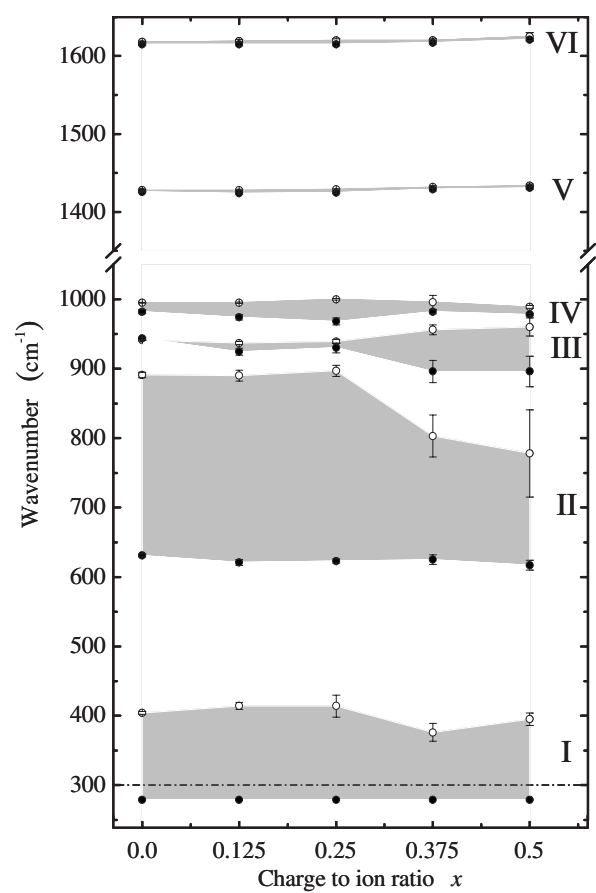

FIG. 8. Dependencies of the $\omega_{\mathrm{TO}, \mathrm{i}}$ and $\omega_{\mathrm{LO}, \mathrm{i}}$ frequencies on the intercalated charge density $x$. The dash-dotted horizontal line indicates the limit of the accessible experimental spectral range.

equivalent to a decrease in the oscillator strength of the stretching $\mathrm{O}-\mathrm{W}-\mathrm{O}$ mode and represents an important quantitative evidence of the interaction of the intercalated charge with the optical phonon structure of amorphous tungsten oxide. The softening of a mode corresponds to a weakening of the chemical bonds reflected by the reduction in the TOmode frequency of the corresponding vibration, whereas a decrease in the polarity of a vibration is associated with the decrease in density of the corresponding bond. When a sixfold ionized tungsten atom is reduced to a fivefold ionized state, it is expected that a reduction in the polarity of these bonds takes place in the $\mathrm{O}-\mathrm{W}-\mathrm{O}$ assigned mode and no softening of the stretching $\mathrm{O}-\mathrm{W}-\mathrm{O}$ should occur.

\section{Bands III and IV}

The vibrational origin of bands III and IV was assigned to terminal $\mathrm{W}=\mathrm{O}$ groups. ${ }^{25} \mathrm{We}$ observe here for $x=0$ that the contribution of band III to the DF is almost negligible; however, with increasing charge intercalation this mode evolves indicative for an increase in $\mathrm{W}=\mathrm{O}$ bonds. In contrast, band IV remains practically unaltered during the intercalation process. Our finding is congruent with observations by Daniel et $a l .{ }^{25}$ who suggested formation of terminal $\mathrm{W}=\mathrm{O}$ groups formed at the film-ambient or film-electrode interfaces of the film. From our IR phonon model calculations we cannot differentiate whether such terminal groups have formed at the film interfaces or else if $\mathrm{W}=\mathrm{O}$ bonds have formed within the film. We argue here that upon extraction of oxygen and intercalation of electrons, $\mathrm{W}-\mathrm{O}-\mathrm{W}$ bonds are broken producing double-bonded $\mathrm{W}=\mathrm{O}$ sites and reduced-oxidation state tungsten sites with attached electrons thereby forming polarons. Because the polaron absorption detected in the 
NIR-VIS DF and discussed in Sec. IV C is a volume property of the film, we conclude that the $\mathrm{W}=\mathrm{O}$ bonds form within the films.

\section{Hydroxyl group vibrations}

Moisture absorption in tungsten oxide originates mainly from the acidic solution employed for the charge intercalation and is typically present. ${ }^{24,34}$ Water has known stretching and bending vibrations of its molecule near 1616, 2780, 3234 , and $3478 \mathrm{~cm}^{-1}$. The small-polarity bands at 1425 and $1620 \mathrm{~cm}^{-1}$ are assigned to hydroxyl group vibrations. Such are obviously present within the films remaining from the chemical coloration process and from further aging upon exposure to normal ambient conditions. ${ }^{34,68}$ We note that modes at 3234 and $3478 \mathrm{~cm}^{-1}$ were not observed here. Incorporation of additional $\mathrm{O}-\mathrm{H}$ bonds during the intercalation process would drastically increase the oscillator amplitudes in the model DF obtained for the intercalated states. As seen in Fig. 8 the small-polarity bands at 1425 and $1620 \mathrm{~cm}^{-1}$ remain practically unaltered upon charge intercalation, which indicates that additional $\mathrm{O}-\mathrm{H}$ bonds are not formed. The broadening parameters for the hydroxyl bands increase slightly upon charge intercalation and which causes the apparent increase in the hydroxyl absorption bands in Figs. 6 and 7. Note, however, the logarithmic scale for both figures, which distorts visual judgment increase in the small contributions from the hydroxyl groups in this presentation.

We conclude from our observations that the oxygenextraction model is relevant for the polaron formation mechanism in amorphous tungsten oxide films. These observations are the experimental evidence of previous firstprinciples pseudopotential calculations within the localdensity approximation, where it was found that an oxygen vacancy in $\mathrm{WO}_{3}$ gives rise to donorlike defect states. ${ }^{69}$ Upon charge intercalation new $\mathrm{W}=\mathrm{O}$ bonds are created, whereas $\mathrm{W}-\mathrm{O}-\mathrm{W}$ bonds are removed. An intercalated electron forms a polaron by changing the oxidation number of a $\mathrm{W}^{6+}$ site to $\mathrm{W}^{5+}$ (or $\mathrm{W}^{5+}$ site to $\mathrm{W}^{4+}$ ). The extracted oxygen leaves two tungsten sites with unsaturated bonds; one of which is adopted by forming a double $\mathrm{W}=\mathrm{O}$ bond, and the other forms a $\left[\mathrm{W}^{5+}, e\right]$ or $\left[\mathrm{W}^{4+}, e\right]$ polaron. The hydrogenintercalation model assumes that hydrogen is stored in the $A$ place of the perovskitelike $\mathrm{WO}_{3}$ structure creating additional $\mathrm{O}-\mathrm{H}$ bonds and $\left[\mathrm{W}^{5+}, e\right]$ or $\left[\mathrm{W}^{4+}, e\right]$ polarons. Because the $\mathrm{O}-\mathrm{H}$ modes remain unchanged in our investigations, we argue that no additional hydrogen is intercalated. We conclude that polaron formation has occurred as described within the oxygen-extraction model.

Finally, we note that for all studied samples, the absence of free-charge carriers was a common characteristic, which was revealed by the absence of a Drude-type oscillator $\left(\omega_{\mathrm{TO}}=0\right)$ in our best-match model DF. IRSE has been recently used as precise means to study the free-charge-carrier properties in semiconductor thin films. Existence of an insulator-to-metal transition in amorphous tungsten oxide films was previously concluded by Crandall and Faughnan ${ }^{37}$ from electrical conductivity measurements. ${ }^{37}$ The authors attributed a strong increase in conductivity to the transformation of the polarons to a metal-like conductivity and which was observed for intercalated charge-density ratios above a critical value of $x_{c}=0.32$. Accordingly, the samples studied here with $x=0.5$ should well reflect this transition. The absence of free-charge-carrier contributions to the IR response here might find an explanation by a large effective mass of the polarons. The detection limits for the determination of free-charge-carrier parameters depend on the effective carrier mass $m_{\text {eff }}$, the carrier mobility $\mu$, and the layer thickness $d .^{70}$ Assuming a charge-carrier mobility of $20 \mathrm{~cm}^{2} / \mathrm{V} \mathrm{s}$, which renders a typical conductivity of amorphous materials, and a free-charge-carrier effective mass of $m_{\mathrm{eff}}=1.75,{ }^{71}$ our estimated detection limits for free-charge-carrier concentration is $\sim 3 \times 10^{17} \mathrm{~cm}^{-3} .38,39$ These values correspond to a resistivity of $\sim 1 \Omega \mathrm{cm}$ and which is higher than the value reported by Crandall and Faughnan. ${ }^{37}$ The electrical conductivity reported by Crandall and Faughnan ${ }^{37}$ should produce a measurable Drude absorption, unless the actual effective mass of the polarons is much larger than $m_{\mathrm{eff}}=1.75$. In fact, the polaron energies plotted in Fig. 4 reveal that the polarons are still bound charge carriers. While their energies begin to decrease, the associated bond charge cloud is still present dressing the charge carriers with large effective mass. Accordingly, no free-charge-carrier Drude term is detected in our investigation.

\section{SUMMARY}

We obtained the evolution of the polaron and lattice mode properties in amorphous tungsten oxide thin films by spectroscopic ellipsometry. Analysis of the dielectric function in the infrared to ultraviolet spectral regions as a function of the intercalated charge density resulted in parametric physical model functions. We observed a strong increase in the polaron absorption in the visible spectral range, a decrease in the infrared $\mathrm{W}-\mathrm{O}$ bond polarity, and an increase in the $\mathrm{W}$ $=\mathrm{O}$ bond polarity upon charge intercalation. Our findings support the oxygen-extraction model as the polaron formation mechanism in tungsten oxide in accordance with previous first-principles pseudopotential calculations. We suggested polaron formation by oxygen-related defect generation as the origin for the coloration mechanism in tungsten oxide. We further discuss possible evidence for very large effective mass of the polarons within the insulator-tometal transition regime.

\section{ACKNOWLEDGMENTS}

We gratefully acknowledge support by NSF within MRSEC Q-SPINS (M.S.) and by NSF-SGER (E.S.), by the J. A. Woollam Foundation, and by startup funds from $\mathrm{CoE}$ at UNL (M.S. and E.S.). 
*http://ellipsometry.unl.edu

†schubert@engr.unl.edu

${ }^{1}$ C. F. Klingshirn, Semiconductor Optics (Springer-Verlag, Berlin, 1995).

${ }^{2}$ J. S. Hale, M. DeVries, B. Dworak, and J. A. Woollam, Thin Solid Films 313-314, 205 (1998).

${ }^{3}$ M. J. DeVries, C. Trimble, T. E. Tiwald, D. W. Thompson, J. A. Woollam, and J. S. Hale, J. Vac. Sci. Technol. A 17, 2906 (1999).

${ }^{4}$ E. B. Franke, C. L. Trimble, M. Schubert, J. A. Woollam, and J. S. Hale, Appl. Phys. Lett. 77, 930 (2000).

${ }^{5}$ J. Solis, A. Hoel, V. Lantto, and C. G. Granqvist, J. Appl. Phys. 89, 2727 (2001).

${ }^{6}$ S. K. Deb, Philos. Mag. 27, 801 (1973).

${ }^{7}$ C. M. Lampert, Sol. Energy Mater. Sol. Cells 76, 489 (2003).

${ }^{8}$ A. Azens and C. G. Granqvist, J. Solid State Electrochem. 76, 489 (2003).

${ }^{9}$ M. Kawaminami and T. Hirose, J. Phys. Soc. Jpn. 46, 864 (1979).

${ }^{10} \mathrm{~J}$. B. Goodenough and A. Hamnett, in Semiconductors, LandoltBornstein, New Series, Group III, Vol. 17g, Pt. III, edited by O. Madelung (Springer, Berlin, 1984).

${ }^{11}$ C. G. Granqvist, Handbook of Inorganic Electrochromic Materials (Elsevier, Amsterdam, 1995).

${ }^{12}$ G. M. Ramans, J. V. Gabrusenoks, A. R. Lusis, and A. A. Patmalnieks, J. Non-Cryst. Solids 90, 637 (1987).

${ }^{13}$ A. Hjelm, C. G. Granqvist, and J. M. Wills, Phys. Rev. B 54, 2436 (1996).

${ }^{14}$ L. A. Bursill, J. Solid State Chem. 48, 256 (1983).

${ }^{15}$ M. Kawaminami and T. Hirose, J. Phys. Soc. Jpn. 47, 1733 (1979).

${ }^{16} \mathrm{~K}$. Takano, A. Inouye, S. Yamamoto, M. Sugimoto, M. Yoshikawa, and S. Nagata, Jpn. J. Appl. Phys., Part 1 46, 6315 (2007).

${ }^{17}$ S. K. Deb, Sol. Energy Mater. Sol. Cells 92, 245 (2008).

${ }^{18}$ G. A. Niklasson and C. G. Granqvist, J. Mater. Chem. 17, 127 (2007).

${ }^{19}$ C. G. Granqvist, Sol. Energy Mater. Sol. Cells 60, 201 (2000).

${ }^{20}$ L. Berggren and G. A. Niklasson, Appl. Phys. Lett. 88, 081906 (2006).

${ }^{21}$ E. Ozkan, S.-H. Lee, C. E. Tracy, J. R. Pitts, and S. K. Deb, Sol. Energy Mater. Sol. Cells 79, 439 (2003).

${ }^{22}$ A. Georg, W. Graf, R. Neumann, and V. Wittwer, Solid State Ionics 127, 319 (2000).

${ }^{23}$ A. Georg, W. Graf, R. Neumann, and V. Wittwer, Thin Solid Films 384, 269 (2001).

${ }^{24}$ N. Yoshiike, Y. Mizuno, and S. Kondo, J. Electrochem. Soc. 131, 2634 (1984).

${ }^{25}$ M. F. Daniel, B. Desbat, J. C. Lassegues, and R. Garie, J. Solid State Chem. 73, 127 (1988).

${ }^{26}$ A. Kasic, M. Schubert, T. Frey, U. Köhler, D. J. As, and C. M. Herzinger, Phys. Rev. B 65, 184302 (2002).

${ }^{27}$ V. Darakchieva et al., Phys. Rev. B 75, 195217 (2007).

${ }^{28}$ T. Hofmann, V. Gottschalch, and M. Schubert, Phys. Rev. B 66, 195204 (2002).

${ }^{29}$ C. Bundesmann, A. Rahm, M. Lorenz, M. Grundmann, and M. Schubert, J. Appl. Phys. 99, 113504 (2006).

${ }^{30}$ M. F. Saenger, T. Höing, T. Hofmann, and M. Schubert, Phys. Status Solidi A 205, 914 (2008).

${ }^{31} \mathrm{~K}$. von Rottkay, N. Ozer, M. Rubin, and T. Richardson, Thin
Solid Films 308-309, 50 (1997).

${ }^{32}$ M. Merz, J. Eisenmenger, B. Heinz, and P. Ziemann, Phys. Rev. B 66, 184102 (2002).

${ }^{33}$ J. Lehan, P. Yu, D. Backfisch, and J. Chambers, J. Appl. Phys. 92, 3608 (2002).

${ }^{34}$ A. Antonaia, M. C. Santoro, G. Fameli, and T. Polichetti, Thin Solid Films 426, 281 (2003).

${ }^{35}$ B. Johs, C. M. Herzinger, J. H. Dinan, A. Cornfeld, and J. D. Benson, Thin Solid Films 313-314, 137 (1998).

${ }^{36}$ E. B. Franke, C. L. Trimble, J. S. Hale, M. Schubert, and J. A. Woollam, J. Appl. Phys. 88, 5777 (2000).

${ }^{37}$ R. S. Crandall and B. W. Faughnan, Phys. Rev. Lett. 39, 232 (1977).

${ }^{38}$ A. Kasic, M. Schubert, S. Einfeldt, D. Hommel, and T. E. Tiwald, Phys. Rev. B 62, 7365 (2000).

${ }^{39}$ M. Schubert, Infrared Ellipsometry on Semiconductor Layer Structures: Phonons, Plasmons, and Polaritons (Springer, Berlin, 2004).

${ }^{40}$ Handbook of Ellipsometry, edited by H. G. Tompkins and E. A. Irene (William Andrew, Highland Mills, 2004).

${ }^{41}$ M. Schubert, Ann. Phys. 15, 480 (2006).

${ }^{42}$ R. M. A. Azzam and N. M. Bashara, Ellipsometry and Polarized Light (North-Holland, Amsterdam, 1984).

${ }^{43}$ G. E. Jellison, Jr., Thin Solid Films 313-314, 33 (1998).

${ }^{44}$ T. Hofmann, G. Leibiger, V. Gottschalch, I. Pietzonka, and M. Schubert, Phys. Rev. B 64, 155206 (2001).

${ }^{45}$ M. G. Stachiotti, F. Corà, C. R. A. Catlow, and C. O. Rodríguez, Phys. Rev. B 55, 7508 (1997).

${ }^{46}$ C. Kittel, Introduction to Solid State Physics (Wiley, New York, 1976).

${ }^{47}$ C. R. Pidgeon, Handbook on Semiconductors (North-Holland, Amsterdam, 1980), Vol. 2.

${ }^{48}$ P. Y. Yu and M. Cardona, Fundamentals of Semiconductors (Springer, Berlin, 1996).

${ }^{49}$ C. M. Wolfe, N. Holonyak, and G. E. Stillmann, Physical Properties of Semiconductors (Prentice-Hall, Englewood Cliffs, NJ, 1989).

${ }^{50}$ F. Gervais and B. Piriou, J. Phys. C 7, 2374 (1974).

${ }^{51}$ M. Schubert, T. E. Tiwald, and C. M. Herzinger, Phys. Rev. B 61, 8187 (2000).

${ }^{52}$ L. Ottaviano, L. Lozzi, M. Passacantando, and S. Santucci, Surf. Sci. 73, 475 (2001).

${ }^{53}$ D. W. Bullett, Solid State Commun. 46, 575 (1983).

${ }^{54}$ H. G. Reik and D. Heese, J. Phys. Chem. Solids 28, 581 (1967).

${ }^{55}$ O. F. Schirmer, V. Wittwer, G. Baur, and G. Brandt, J. Electrochem. Soc. 124, 749 (1977).

${ }^{56}$ K. von Rottkay, M. Rubin, and S. J. Wen, Thin Solid Films 306, 10 (1997)

${ }^{57}$ E. Pascual, J. Martí, E. Garcia, E. Canillas, and E. Bertran, Thin Solid Films 313-314, 682 (1998).

${ }^{58}$ A. Antonaia, T. Polichetti, M. Addonizio, S. Aprea, C. Minarini, and A. Rubino, Thin Solid Films 354, 73 (1999).

${ }^{59}$ J. Ederth, A. Hoel, G. A. Niklasson, and C. G. Granqvist, J. Appl. Phys. 96, 5722 (2004).

${ }^{60}$ R. Synowicki, Thin Solid Films 313-314, 394 (1998).

${ }^{61}$ S. Lee, H. Cheong, J. Zhang, A. Mascarenhas, D. Benson, and S. Deb, Appl. Phys. Lett. 74, 242 (1999).

${ }^{62}$ B. Heinz, M. Merz, P. Widmayer, and P. Ziemann, J. Appl. Phys. 90, 4007 (2001).

${ }^{63}$ M. Burdis, J. Siddle, R. Batchelor, and J. Gallego, Proc. SPIE 
2531, 11 (1995).

${ }^{64}$ Equation (10) is valid for unsaturated conditions. Site-saturation models for the optical efficiency of tungsten oxide-based devices were developed by M. Denesuk and D. R. Uhlmann, J. Electrochem. Soc. 143, L186 (1996) and consider blocking of polaron transitions in saturation regimes; a generalization for multiple polaron transitions is derived by L. Berggren and G. A. Niklasson, Appl. Phys. Lett. 88, 081906 (2006).

${ }^{65}$ T. Yoshimura, J. Appl. Phys. 57, 911 (1985).

${ }^{66}$ L. Berggren, A. Azens, and G. A. Niklasson, J. Appl. Phys. 90, 1860 (2001).
${ }^{67}$ J. Humlíček, R. Henn, and M. Cardona, Phys. Rev. B 61, 14554 (2000).

${ }^{68}$ M. F. Daniel, B. Desbat, J. C. Lassegues, B. Gerand, and M. Figlarz, J. Solid State Chem. 67, 235 (1987).

${ }^{69}$ S. Zh. Karazhanov, Y. Zhang, L.-W. Wang, A. Mascarenhas, and S. Deb, Phys. Rev. B 68, 233204 (2003).

${ }^{70}$ A. Kasic, M. Schubert, S. Einfeldt, D. Hommel, and T. E. Tiwald, Phys. Rev. B 62, 7365 (2000).

${ }^{71}$ J. M. Berak and M. J. Sienko, J. Solid State Chem. 2, 109 (1970). 